\title{
Numerical Experimentation to obtain the Laguerre polynomial from average value of all characteristic polynomials of Hermitian Random Matrices
}

\author{
Gonzalez, H.E., and Carmona, L., J.J. \\ Information Technology Department, ININ, ITTLA
}

\begin{abstract}
Although the behavior of all subatomic particles is inherently probabilistic, Schrodinger's equation does not itself contain any probabilities. In this work the Authors reinterprets the Schrodinger Equation; to find in it the randomness that was hidden and that was overlooked by Schrodinger himself. From the generation of Hermitian random matrices and their corresponding characteristic polynomials, the Authors concludes that the radial part solution of the Schrodinger equation for the Hydrogen Atom, namely Laguerre Polynomial, is obtained from the average value of all characteristic polynomials. This is how in this work it is made clear that the deterministic method to obtain the Laguerre Polynomial through the Rodrigues Formula is equivalent to the probabilistic method proposed by the Authors.
\end{abstract}

Index Terms-Characteristic Polynomials, Hermitian Random Matrices, Laguerre Polynomial, Schrodinger Equation of Hydrogen Atom.

\section{INTRODUCTION}

The Laguerre Polynomial is obtained from the Rodrigues Formula [1]-[7]. This way of getting the polynomial, it can be classified as a deterministic method, that is, it does not use probabilities to be able to get your mathematical expression. It has great importance in Quantum Mechanics, especially in the Analytical solution of the Schrodinger Equation for the Hydrogen atom.

From a book published by the Author, a new method is postulated to calculate the Polynomial Characteristic of a Matrix through Principal Minors. This New Method was coded in MatLab language to be able to handle complex numbers, following the instructions indicated in [6].

After deeply studying Quantum Theory, it was thought about the possibility of obtaining the radial part solution of the Schrodinger Equation for the Hydrogen Atom, and that is how it was proposed to generate randomly a certain number of times, Hermitian Matrices of sizes ranging from 1 to 6 and whose coefficients, both the real ones and those that accompany the imaginary number, are random numbers of a Normal Probability Density Distribution with zero mean and one variance. The cases for sizes 1 to 6 are illustrated in this work.

Manuscript received: 25 August 2021

Manuscript received in revised form: 20 September 2021

Manuscript accepted: 6 October 2021

Manuscript Available online: 15 October 2021
The size of the randomly generated Standard Normal Hermitian matrix corresponds to the Degree of the Laguerre Polynomial, in such a way that for Degree 1, 5 complex values are generated random matrices, which correspond to random matrices of size one and 5 characteristic polynomials are obtained, one for each matrix, to be added later and of the five, only one is obtained, whose coefficients will be the sums obtained divided by five and rounding to the nearest integer, to obtain the averages of the coefficients of the only Characteristic Polynomial that remains when performing these operations and that, to the Author's surprise, is none other than Laguerre's Polynomial. This is illustrated in next section.

\section{NUMERIC EXPERIMENTATION}

For $N=1$ (Size Matrix 1) and $n=5$ (Sample Size)

Table 1. Generated Normal Random Numbers and their Characteristic

\begin{tabular}{|l|l|l|}
\hline No. & \multicolumn{1}{|c|}{$\begin{array}{c}\text { Generated Normal Random } \\
\text { Numbers }\end{array}$} & \multicolumn{1}{c|}{$\begin{array}{c}\text { Characteristic } \\
\text { Polynomial }\end{array}$} \\
\hline 1 & 0.93481 & $-\mathrm{x}+0.93481$ \\
\hline 2 & 0.43038 & $-\mathrm{x}+0.43038$ \\
\hline 3 & 0.41366 & $-\mathrm{x}+0.41366$ \\
\hline 4 & 1.95870 & $-\mathrm{x}+1.95870$ \\
\hline 5 & 2.55820 & $-\mathrm{x}+2.55820$ \\
\hline $\bar{x}$ & $6.29575 / 5=1.25915$ & $\begin{array}{l}(-5 \mathrm{x}+6.29575) / 5=-\mathrm{X}+1 \\
(\text { Rounded })\end{array}$ \\
\hline
\end{tabular}

For $N=2$ (Size Matrix 2) y $n=5$ (Sample Size)

Table 2. Generated Normal Random Numbers and their Characteristic

\begin{tabular}{|c|c|c|}
\hline No & Generated Normal Random Numbers & $\begin{array}{l}\text { Characteristic } \\
\text { Polynomial }\end{array}$ \\
\hline 1 & $\left(\begin{array}{cc}1.42317 & 0.70066-0.67644 i \\
0.70066+0.67644 i & 0.71935\end{array}\right)$ & $x^{2}-2142523 x+0.0753260$ \\
\hline 2 & $\left(\begin{array}{cc}3.33339 & -0.21918+1.06537 i \\
-0.21918-1.06537 i & 2.38785\end{array}\right)$ & $x^{2}-5.721277 x+6.77654$ \\
\hline 3 & $\left(\begin{array}{cc}1.83959 & 0,11016+1.77489 i \\
0,11016-1.77489 i & 1.80702\end{array}\right)$ & $x^{2}-3.64660 x+0.16190$ \\
\hline 4 & $\left(\begin{array}{cc}1.9826 & 21796+0.6259 i \\
2.1796-0.6259 i & 4.3797\end{array}\right)$ & $x^{2}-6.36220 x+3.54050$ \\
\hline 5 & $\left(\begin{array}{cc}0.94561 & -0.40675-0.511094 i \\
-0.40675+0.511094 i & 0.50349\end{array}\right)$ & $x^{2}-1.44910 x+0.04960$ \\
\hline$\overline{\bar{x}}$ & 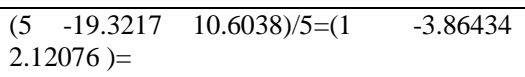 & $x^{2}-4 x+2$ (Rounded) \\
\hline
\end{tabular}


ISSN: 2277-3754

ISO 9001:2008 Certified

International Journal of Engineering and Innovative Technology (IJEIT)

Volume 11, Issue 3, September 2021

As the Laguerre Polynomial is obtained from the generation of Standardized Normal Random Hermitian matrices, there are several solutions, for example this would be another:

For $N=2$ (Size Matrix 2) and $n=5$ (Sample Size)

Table 3. Generated Normal Random Numbers and their Characteristic

\begin{tabular}{|c|c|c|}
\hline No & Generated Normal Random Numbers & $\begin{array}{l}\text { Characteristic } \\
\text { Polynomial }\end{array}$ \\
\hline 1 & $\begin{array}{cc}0.22287 & 0.19103+0,08774 i \\
0.19103-0.08774 i & 159031\end{array}$ & $x^{2}-1.81318 x+0.31024$ \\
\hline 2 & $\left.\begin{array}{cc}3.1102 & 1.0806-1.4893 i \\
1.0806+1.4893 i & 1.8104\end{array}\right)$ & $2 x+2.24506$ \\
\hline 3 & $\begin{array}{cc}481954 & -0,43283+172809 \\
-0,43283-1,72809 i & 1,13106 \\
\end{array}$ & $x^{2}-5.95060 x+2.27760$ \\
\hline 4 & $\left(\begin{array}{cc}0.60313 & -0,11772-0.51754 i \\
-0.11772+0.51754 i & 0.92411\end{array}\right)$ & $x^{2}-1.52720 x+0.27560$ \\
\hline 5 & $\left(\begin{array}{cc}11380 & 1.4127+0.8997 i \\
1.4127-0.8997 i & 48611\end{array}\right)$ & $x^{2}-5.99910 x+2.72670$ \\
\hline$\overline{\bar{x}}$ & $\begin{array}{l}(5-20.2108 \quad 7.8352) / 5=(1 \quad-4.04216 \\
1.56704)=\end{array}$ & $\begin{array}{l}x^{2}-4 x+2 \text { (Rounde } \\
\text { d) }\end{array}$ \\
\hline
\end{tabular}

And despite being a group of values different from the previous ones, it will always have the same expected value.

For N=3 (Size Matrix 3) y $n=60$ (Sample Size)

Table 4. Generated Normal Random Numbers and their Characteristic

\begin{tabular}{|c|c|c|c|c|}
\hline \multirow{2}{*}{\multicolumn{3}{|c|}{$\begin{array}{c}\text { For } N=3 \text { (Size Matrix 3) y } n=60 \text { (Sample Size) } \\
\text { Table 4. Generated Normal Random Numbers and their } \\
\text { Characteristic }\end{array}$}} & \multicolumn{2}{|r|}{ Generated Normal Random Numbers } \\
\hline & & & 1 & 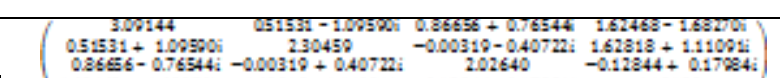 \\
\hline No. & Generated Normal Random Numbers & $\begin{array}{l}\text { Characteristic } \\
\text { Polynomial }\end{array}$ & & 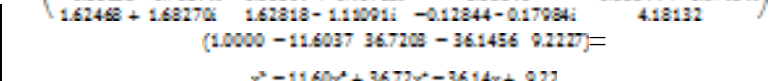 \\
\hline 1 & 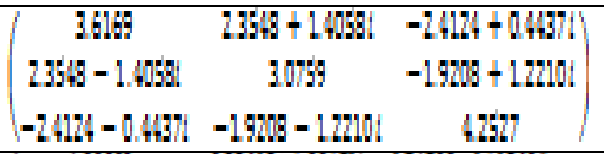 & $-x^{x}+10.9655 x^{x}-20.9696$ & $2000^{\circ}$ & 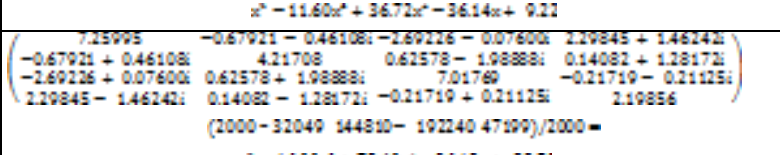 \\
\hline 2 & 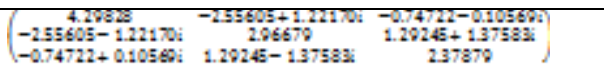 & $-x^{x}+9.6439 x^{x}-17.8756 x$ & +20352 & 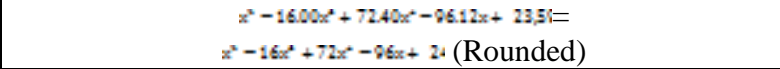 \\
\hline & & & 2100 & 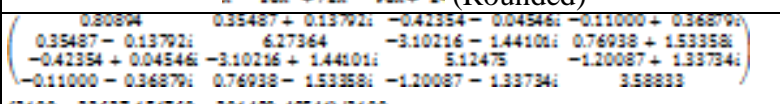 \\
\hline 59 & 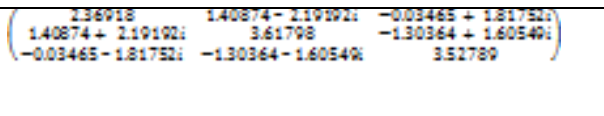 & $-x^{x}+9515 x^{x}-15323 x+4$ & 2.692 & 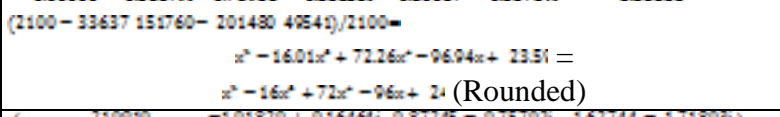 \\
\hline & & & 2200 & 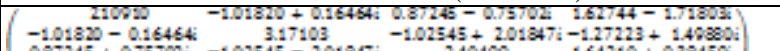 \\
\hline 60 & 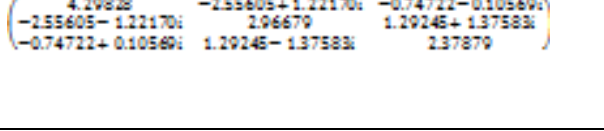 & $-x^{2}+5126 x^{x}-6185 x+d$ & 675 & 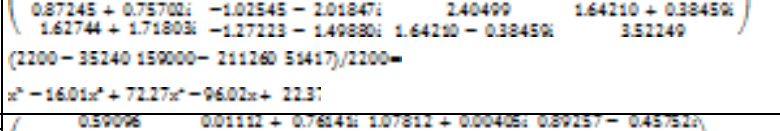 \\
\hline$\overline{\bar{x}}$ & 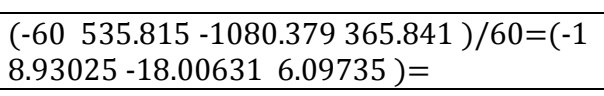 & $\begin{array}{l}-x^{x}+9 x^{x}-19 x+6 \\
\text { (Rounded) }\end{array}$ & 2300 & 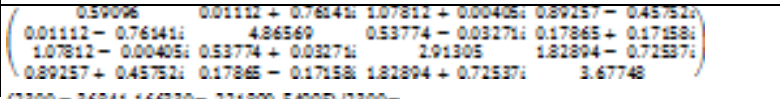 \\
\hline & As the Laguerre Polynomial is ol & & & 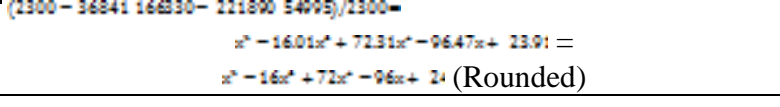 \\
\hline
\end{tabular}
of Standardized Normal Random Hermitian matrices, there are several solutions, for example this would be another:

For $N=3$ (Size Matrix 3) and $n=100$ (Sample Size)

Table 5. Generated Normal Random Numbers and their Characteristic

\begin{tabular}{|c|c|c|}
\hline No. & Generated Normal Random Numbers & $\begin{array}{l}\text { Characteristies the Laguerre Polynomial is obtained } \\
\text { Polynomigeneration of Standardized Normal Random }\end{array}$ \\
\hline 1 & 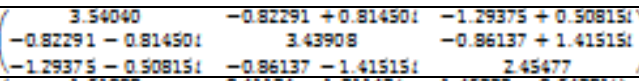 & $\begin{array}{l}-x^{x}+9.436 \text { mateices, thene } \text { are several solutions, for example } \\
\text { be another. }\end{array}$ \\
\hline 20 & 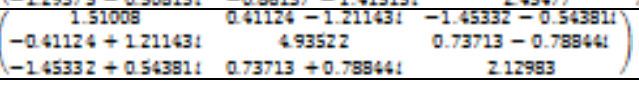 & 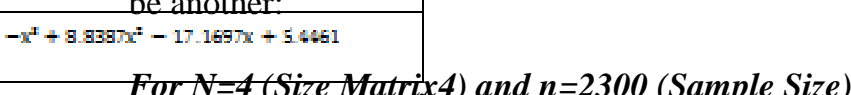 \\
\hline
\end{tabular}

$\left(\begin{array}{llll}-20 & 176.774 & 343.394 & -108.922\end{array}\right) / 20=\left(\begin{array}{ll}-1 & 8.8387\end{array}\right.$ $-17.16975 .4461) / 20=$

$40 \quad\left(\begin{array}{ccc}5.01263 & 4.36961+0.173031 & -0.67628-2.3033699 \\ 0.36941-0.173031 & 333368 & 0.51533-2.719699\end{array}\right)$ 4-0.67628 + 2.303361 051593 +2.71969: 256179

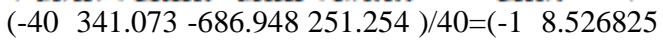
$-17.17376 .28135) / 40=$

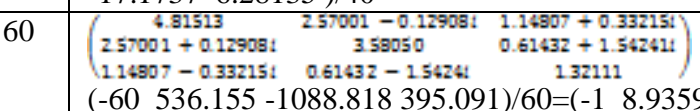
$-18.146966 .58485) / 60=$

\begin{tabular}{|c|c|c|}
\hline 80 & 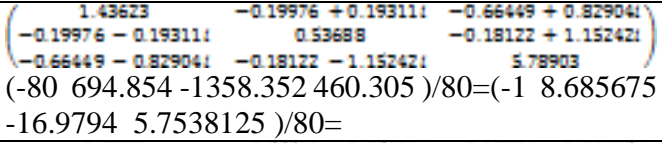 & $-x^{x}+13655675 x^{2}-$ \\
\hline 100 & 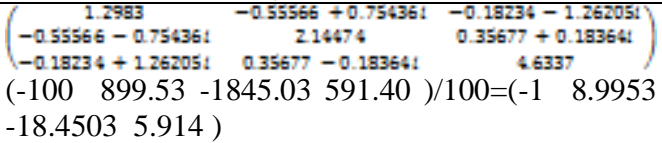 & \\
\hline
\end{tabular}

* Unlike the previous table, this means that the indicated average polynomial is the one obtained based on the number of matrices generated up to that moment, as indicated in the last line of each row of the second column.

\section{For $N=4$ (Size Matrix4) and $n=2300$ (Sample Size)}

Table 6. Generated Normal Random Numbers and their Characteristic Generated Normal Random Numbers

Like the previous table, this means that the indicated average polynomial is the one obtained based on the number of matrices generated up to that moment, as indicated in the last line of each row of the second column. 
ISSN: 2277-3754

\section{ISO 9001:2008 Certified}

International Journal of Engineering and Innovative Technology (IJEIT)

Volume 11, Issue 3, September 2021

Table 7. Generated Normal Random Numbers and their

Characteristic

\begin{tabular}{|c|c|}
\hline No. & Generated Normal Random Numbers \\
\hline 1 & 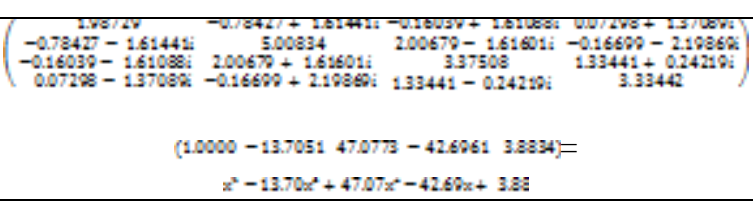 \\
\hline 1200 & 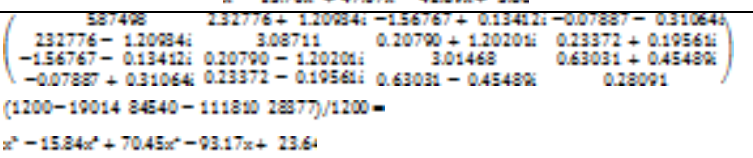 \\
\hline 1500 & 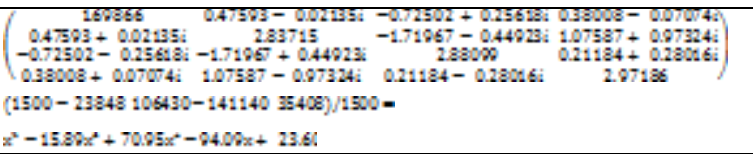 \\
\hline 1800 & 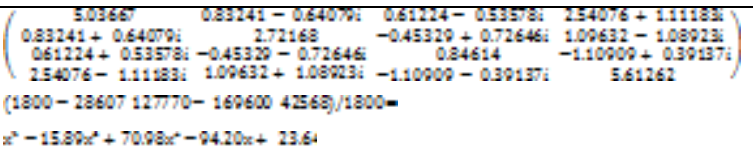 \\
\hline 2100 & 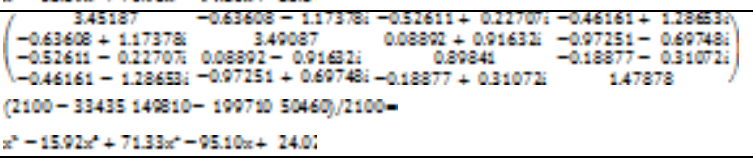 \\
\hline 2300 & 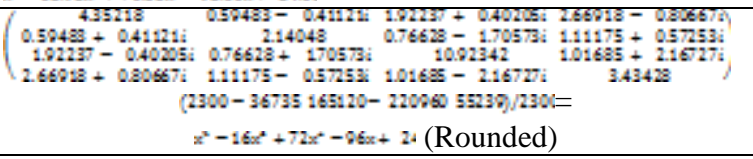 \\
\hline
\end{tabular}

For $N=5$ (Size Matrix5) and $n=6500$ (Sample Size)

Table 8. Generated Normal Random Numbers and their Characteristic

\begin{tabular}{|c|c|}
\hline No. & Generated Normal Random Numbers \\
\hline 1 & 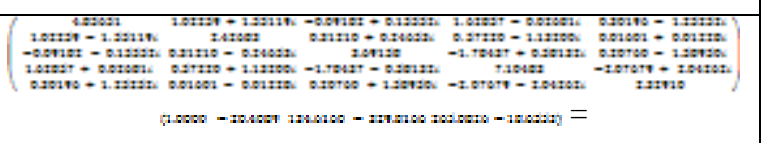 \\
\hline 5000 & 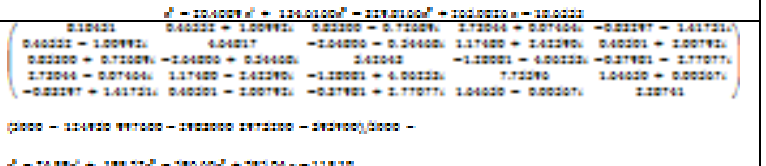 \\
\hline 6000 & 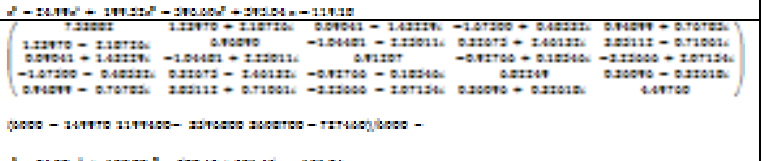 \\
\hline 6500 & 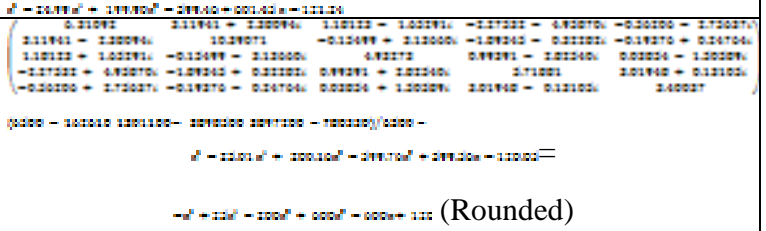 \\
\hline
\end{tabular}

For $\mathbf{N}=6$ (Size Matrix6) and $\mathbf{n = 6 5 0 0 0}$ (Sample Size)
Table 9. Generated Normal Random Numbers and their

Characteristic

Generated Normal Random Numbers

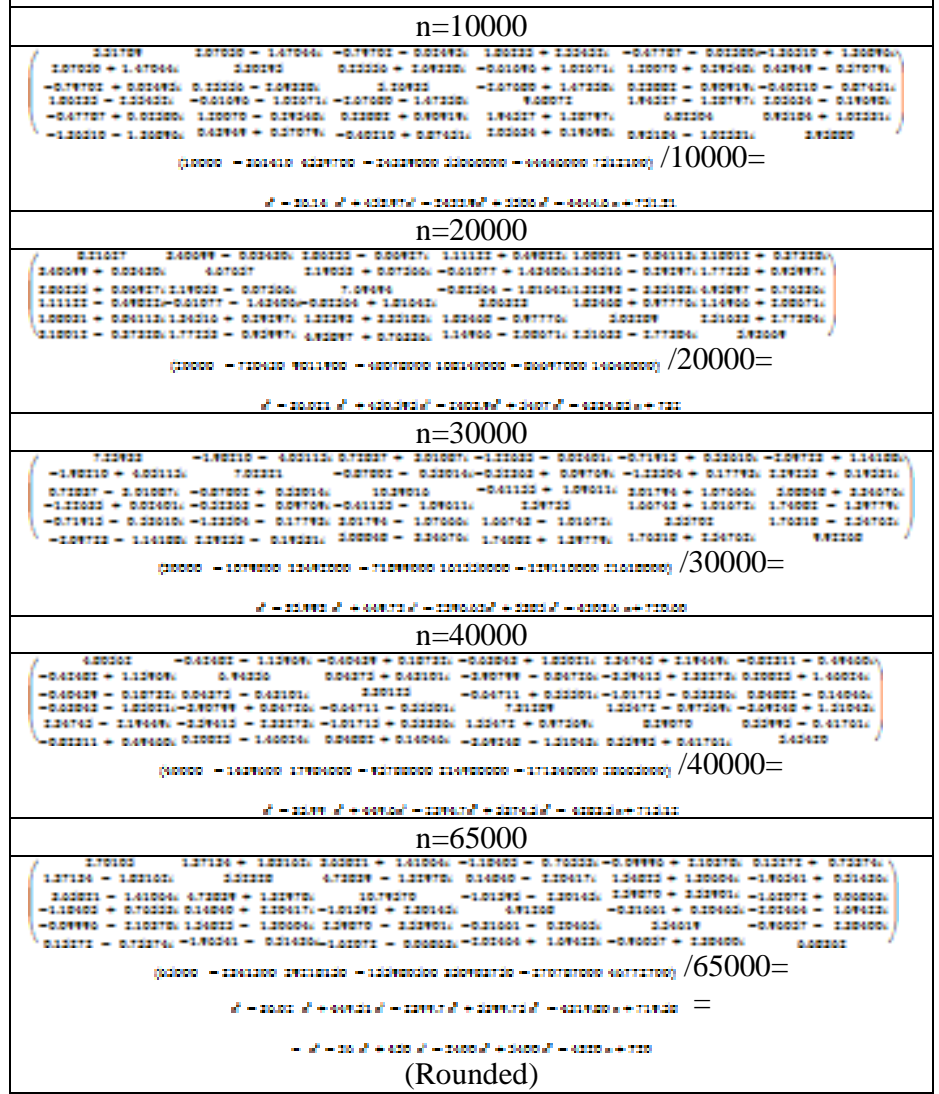

On the other hand, the Laguerre Associated Polynomials are obtained from the derivative of the above:

Table 10.Different parameters value

\begin{tabular}{|l|l|}
\hline \multicolumn{1}{|c|}{$L_{n}(x)$} & \multicolumn{1}{c|}{$L_{n}(x)$} \\
\hline 1 & $\mathbf{0}$ \\
\hline$-\mathbf{x}+\mathbf{1}$ & $\mathbf{- 1}$ \\
\hline$x^{2}-4 x+2$ & $-3 x^{2}+18 x-18$ \\
\hline$-x^{3}+9 x^{2}-18 x+6$ & $4 x^{3}-48 x^{2}+144 x-$ \\
\hline$x^{4}-16 x^{3}+72 x^{2}-96 x$ & \\
\hline$-x^{5}+25 x^{4}-200 x^{3}+6$ & $-5 x^{4}+100 x^{3}-600 x$ \\
& \\
\hline$x^{6}-36 x^{5}+450 x^{4}-24$ & $6 x^{5}-180 x^{4}+1800 x^{3}$ \\
& \\
\hline
\end{tabular}

\section{QUANTUM MECHANICS}

It is convenient to have the Laguerre Associated Polynomials, referring to the quantum numbers [7], as follows: The superscript indicates derivation and the subscript is added to the previous one to obtain the degree of 
ISSN: 2277-3754

ISO 9001:2008 Certified

International Journal of Engineering and Innovative Technology (IJEIT)

Volume 11, Issue 3, September 2021

the original Laguerre polynomial, multiplied by minus one, The solution to the Differential Equation above is: (where

obtaining the following table :

Table 11.Different Parameters value

\begin{tabular}{|l|l|l|}
\hline $\mathrm{n}$ & 1 & $L_{x-1-1}^{21+2}(x)$ \\
\hline 1 & 0 & $L_{0}^{2}(x)=1$ \\
\hline 2 & 0 & $L_{2}^{2}(x)=-2 x+4$ \\
\hline 2 & 1 & $L_{0}^{2}(x)=6$ \\
\hline 3 & 0 & $L_{2}^{2}(x)=3 x^{2}-18 x+18$ \\
\hline 3 & 1 & $L_{2}^{2}(x)=-24 x+96$ \\
\hline 3 & 2 & $L_{0}^{3}=120$ \\
\hline
\end{tabular}

Next, it is shown how the mathematical expressions that represent the solution of the Schrodinger Equation are obtained using the previous associated Laguerre polynomials:

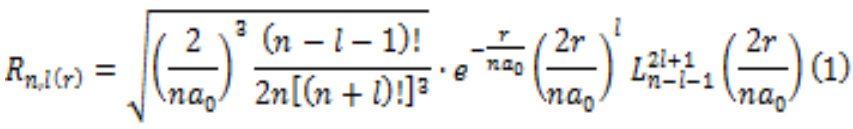

Where $r$ is the distance from the electron to the nucleus of the Hydrogen atom and $a_{0}$ is the Bohr radius. Expressions taken as an example are obtained for $\mathrm{n}=1,2,3 \ldots$ and $1=$ $0,1,2, \ldots,(n-1)$, as illustrated in the following table:

Table 12. Different Parameters value

\begin{tabular}{|c|c|c|}
\hline $\mathrm{n}$ & 1 & 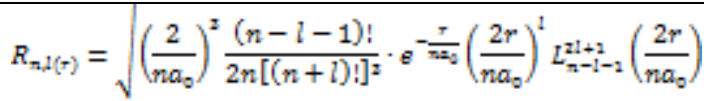 \\
\hline 1 & 0 & $R_{1, p(r)}=2 a_{a}^{-\frac{1}{2}}-a^{-\frac{r}{a_{2}}}-L_{a}^{2}\left(\frac{2 r}{a_{0}}\right)$ \\
\hline 2 & 0 & 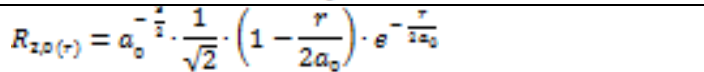 \\
\hline 2 & 1 & 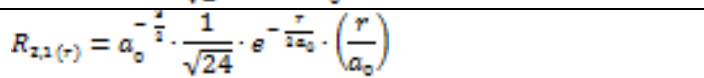 \\
\hline 3 & 0 & $R_{x p(r)}=\frac{2}{\sqrt{27}}-a_{0}^{-\frac{1}{2}}-e^{-\frac{\gamma}{2 x_{a}}} \cdot\left[1-\frac{2 r}{3 a_{0}}+\frac{2}{27} \cdot \frac{\gamma^{2}}{a_{0}^{2}}\right]$ \\
\hline 3 & 1 & 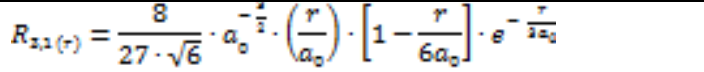 \\
\hline 3 & 2 & 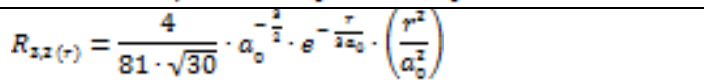 \\
\hline
\end{tabular}

It can be seen that the Laguerre polynomial constitutes the fundamental solution to the equation and from there the associates are derived.

The allowed energies $E_{n}$ of the electron in the $\mathrm{n}$ different levels of the Hydrogen atom are obtained from the previous equation:

$\frac{\mathrm{d}^{2}}{\mathrm{~d} r^{2}}(\mathrm{rR}(\mathrm{r}))-\frac{2 \mathrm{~m}_{\mathrm{e}}}{\hbar^{2}}\left[U(r)+\frac{l(l+1) h^{2}}{2 \mathrm{~m}_{\mathrm{e}} r^{2}}-E\right](r \mathbb{R}(r))=0$

Where:U(r) $=-\frac{1}{4 \pi \varepsilon_{0}} \frac{e^{2}}{r}$

Consider for when $1=0$, then $\mathrm{n}=1$, substituting:

$$
\frac{\mathrm{d}^{2}}{\mathrm{~d} r^{2}}\left(\mathrm{rR}_{1,0}(\mathrm{r})\right)+\frac{2 \mathrm{~m}_{\mathrm{g}}}{\hbar^{2}}\left[\frac{1}{4 \pi r 0} \frac{\mathrm{e}^{2}}{\mathrm{r}}+E_{1}\right]\left(r \mathbb{R}_{1,0}(r)\right)=0
$$

$$
\left.A=2 a_{0}^{-\frac{3}{2}}(5)\right) \quad R_{n=1, l=0}(r)=A e^{-\frac{r}{a_{0}}}
$$

Where, $a_{0}$ is a parameter to be determined. Doing the mathematics, substituting the solution in the Equation, this and its derivatives, must convert it into an Identity:

$$
\begin{gathered}
\frac{\mathrm{d}^{2}}{\mathrm{~d} r^{2}}(\operatorname{rR}(\mathrm{r}))=\frac{\mathrm{d}^{2}}{\mathrm{~d} r^{2}}\left(r A e^{-\frac{r}{a_{0}}}\right)=\frac{d}{d r}\left(A e^{-\frac{r}{a_{0}}}+r\left(-\frac{1}{a_{0}} A e^{-\frac{r}{a_{0}}}\right)\right)= \\
=\left(-\frac{1}{a_{0}}\right) A e^{-\frac{r}{a_{0}}}+\left(-\frac{1}{a_{0}}\right) A e^{-\frac{r}{a_{0}}}+r\left(-\frac{1}{a_{0}}\right)^{2} A e^{-\frac{r}{a_{0}}}= \\
=\left(-\frac{2}{a_{0}}\right) A e^{-\frac{\sigma_{0}}{a_{0}}}+r\left(\frac{1}{a_{0}}\right)^{2} A e^{-\frac{r}{a_{0}}} \text { (7) }
\end{gathered}
$$

Applying in (4):

$\left(-\frac{2}{a_{0}}\right) A e^{-\frac{r}{a_{0}}}+r\left(\frac{1}{a_{0}}\right)^{2} A e^{-\frac{r}{a_{0}}}+\frac{2 m_{e}}{h^{2}}\left[\frac{1}{4 \pi \varepsilon_{0}} \frac{e^{2}}{r}+E_{1}\right]\left(r A e^{-\frac{r}{a_{0}}}\right)=0$

Factoring and simplifying:

$$
\begin{aligned}
& \left(-\frac{2}{a_{0}}\right)+r\left(\frac{1}{a_{0}}\right)^{2}+\frac{2 \mathrm{~m}_{\mathrm{e}}}{h^{2}}\left[\frac{1}{4 \pi \varepsilon_{0}} \frac{\mathrm{e}^{2}}{\mathrm{r}}+E_{1}\right] r=0 \\
& \left(\frac{2 \mathrm{~m}_{\mathrm{e}} E_{1}}{\hbar^{2}}+\frac{1}{a_{0}^{2}}\right) \mathrm{r}+\left(\frac{2 \mathrm{~m}_{\mathrm{e}}}{\hbar^{2}} \frac{\mathrm{e}^{2}}{4 \pi \varepsilon_{0}}-\frac{2}{a_{0}}\right)=0
\end{aligned}
$$

For the above equation to be true for all $r$, both terms in parentheses must be identically 0 , which gives us two equations:

$$
\begin{aligned}
& \left(\frac{2 \mathrm{~m}_{\mathrm{e}} E_{1}}{\hbar^{2}}+\frac{1}{a_{0}^{2}}\right)=0 \Rightarrow E_{1}=-\frac{\hbar^{2}}{2 m_{e}} \frac{1}{a_{0}^{2}}(11) \\
& \left(\frac{2 \mathrm{~m}_{\mathrm{e}}}{\hbar^{2}} \frac{\mathrm{e}^{2}}{4 \pi \varepsilon_{0}}-\frac{2}{a_{0}}\right)=0
\end{aligned}
$$

Solving (12) for: (radius of the electron in its ground state)

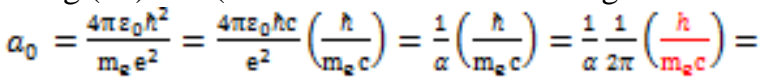

\section{$52.9 \mathrm{pm}$}

Where:

a) A picometer $(\mathbf{p m})=1 \cdot 10^{-} \mathrm{m}$, which is what Niels Bohr found in 1911 with semi-classical calculations applied to the hydrogen atom.

\section{b) Compton wavelength:}

$\alpha=\frac{C^{2}}{\left(C^{2} / N m^{2}\right)(j s)(m / s)}=\frac{N m^{2}}{J m}=\frac{N m}{J}=$ dimensionless

It is seen that it has no units.

Knowing we solve the equation (11):

$$
\begin{aligned}
& E_{1}=-\frac{h^{2}}{2 m_{\theta}} \frac{1}{a_{0}^{2}} \frac{a_{0}^{2}}{\left(\frac{1}{\alpha} \frac{1}{2 \pi}\left(\frac{h}{m_{2} c}\right)\right)^{2}}=-\frac{h^{2}}{2 m_{\theta}}\left(\alpha^{2}\right)(2 \pi)^{2}\left(\frac{m_{\theta} \mathrm{c}}{h}\right)^{2}=-\frac{1}{2} \mathrm{~m}_{\theta}(c \alpha)^{2}= \\
& \left.=\frac{9.11 \times 10^{-71} \mathrm{~kg} \cdot\left(3 \times 10^{8} \mathrm{~m} / \mathrm{s}\right)^{2}}{2} \times\left(\frac{1}{137.04}\right)^{2}=-4.0995 \times 10^{-14}\right] \times\left[5.328 \times 10^{-5}\right]=
\end{aligned}
$$


ISSN: 2277-3754

ISO 9001:2008 Certified

International Journal of Engineering and Innovative Technology (IJEIT)

Volume 11, Issue 3, September 2021

$$
=-2.184 \mathrm{x} 10^{-19} \mathrm{~J} \mathrm{x} \frac{1 \mathrm{eV}}{1.602 \mathrm{x} 10^{-19} \mathrm{~J}}=-13.6 \mathrm{eV}
$$

In general the energies are determined by $\mathrm{n}$ :

$$
E_{n}=\frac{-13.6 e V}{(n)^{2}} \forall n=1,2, \quad 0 \leq l \leq n-1
$$

\section{CONCLUSION}

Authors concludes that the solution of the Schrodinger equation is obtained from the average value of all characteristic polynomials, that is, it demonstrates the following limit numerically:

$$
\lim _{n \rightarrow=\infty}\left(\frac{1}{n} \cdot \sum_{i=1}^{n} \text { PolChar }_{i}^{N}\right)=\text { Pol Lagwes }_{\text {Na }}^{N} \text { (16) }
$$

Where $\mathrm{N}$ is the size of the matrix and $\mathrm{n}$ is the number of random matrices generated, all different, that allow obtaining the limit. Physically, $\mathrm{N}$ would be the energy level of the Hydrogen atom and $n$ the number of wave collapses that would give the position of the electron at a given moment at that level and all those that are less than it.

\section{REFERENCES}

[1] S Altmann, E L Ortiz (eds.), "Mathematics and social utopias in France", Olinde Rodrigues and his times (American Mathematical Society, Providence RI, Pages: 1-13, 2005).

[2] S L Altmann, Hamilton, "Rodrigues, and the quaternion scandal”, Math. Mag. 62 (5) Pages: 291-308, 1989.

[3] H Cheng and K C Gupta, "An historical note on finite rotations", Trans. ASME J. Appl. Mech., 56 (1), Pages: 139-145, 1989.

[4] J J Gray, Olinde Rodrigues', “paper of 1840 on transformation groups”, Arch. Hist. Exact Sci., 21 (4), Pages: 375-385, 1979.

[5] V V Povstenko, "The studies of Rodrigues in kinematic geometry (Ukrainian)", Naturalist and Tech. Issue, 22, Pages: 35-37, 1978.

[6] Pina Garza, E.,Pacheco Quintanilla, M.E.,., "Benjamin Olinde Rodrigues, mathematician and philanthropist, and his influence on Mexican Physics ", Mexican Journal of Physics 57 (1), Pages: 109-113, June 2011.

[7] Gonzalez, H.E., "Characteristic Polynomial Theorem. A new method to obtain the coefficients of the Characteristic Polynomial”, INIS, Mexico, Pages: 1-46, May 2009.

\section{AUTHOR BIOGRAPHY}

H. E. González. He received the PhD in Operations Research at the National Autonomous University of Mexico (UNAM) in 2005. He has been working in research activities for more than twenty years. He has published a book and more than fifteen scientific papers. Presently, he is a full-time researcher at the National Institute of Nuclear Research (ININ) at the Department of Systems.

J.J. Carmona L., He received his B.S. in Electronic Engineering from UAM. Presently, he is a full-time researcher at the National Institute of Nuclear Research (ININ) at the Department of Systems. 